\title{
Quality control test for electronic portal imaging device using QC-3 phantom with PIPSpro
}

\author{
Birendra Kumar Rout ${ }^{1,2}$, Mukka Chandra Shekar'2, Alok Kumar ${ }^{3}$, Kondapalli Kesava Durga Ramesh ${ }^{4}$ \\ ${ }^{1}$ Department of Radiation Physics, Aditya Birla Memorial Hospital, Pune, India \\ ${ }^{2}$ Department of Physics, Jawaharlal Nehru Technological University, Hyderabad, India \\ ${ }^{3}$ Department of Radiation Physics, Mahavir Cancer Sansthan, Patna, India \\ ${ }^{4}$ Department of Radiation Oncology, Navodaya Cancer Institute \& Research Center, Bhopal, India
}

Received July 10, 2014; Revised August 23, 2014; Accepted September 18, 2014; Published Online September 29,2014

\section{Original Article}

\begin{abstract}
Purpose:A Quality control (QC) test suitable for routinely daily use has been established for electronic portal imaging device (EPID) using PIPSpro software, version 4.4. It provides an objective and quantitative test for tolerable image quality on the basis of the high contrast spatial resolution, the contrast-to-noise ratio (CNR) and noise.Methods: The test uses a QC-3 phantom consisting of five sets of high contrast rectangular bar patterns with spatial frequeinces of $0.10,0.20,0.25,0.43$ and 0.75 $\mathrm{lp} / \mathrm{mm}$ using $6 \mathrm{MV}$ and $15 \mathrm{MV}$ photon energy for accquiring high quality images. A "base line" value for the relative square wave modulation transfer function (RMTF), CNR and Noise data was obtained during a one week calibration period and one month test period. Results: Subsequent measurements shows significant deviations from baseline values, resulting in warning messages "potential problems in system performance". The QC test uses high contrast spatial resolution and CNR for the system with acceptable performance. Conclusion: The method provides an automatic, objective, and sensitive measure of the system's imaging performance. This is a useful implementation during acceptance testing, commissioning, and routine qualitycontrol.
\end{abstract}

Keywords: EPID; QC-3 Phantom; PIPSpro; Spatial Resolution; CNR; Noise

\section{Introduction}

Verification of the field placement is an indispensable part of a compressive quality assurance program for radiation oncology. Task group (TG) report ${ }^{1}$ suggested by American Association of Physicists in Medicine (AAPM) recommends for acquiring portal images at least once in a week, which is frequently used in most of the clinical practices. With the recent expansion of electronic portal devices ${ }^{2}$ (EPID), verification is now much simpler and could be carried out on a more frequent basis with the expectation of reducing gross field placement errors and increasing overall treatment accuracy.

However, the effectiveness of an EPID depends mostly on the image quality of the device to verify patient set-up and positioning prior to radiation therapy treatments. ${ }^{3}$ Hence, it is equally important for device itself to maintain in its design and devise quality control (QC) tests to boost better image quality. These tests are essential by the manufacture at design, manufacture, operation, installation and by the user, all the way through the lifetime of the equipment. Nevertheless, as these systems are the part of regular clinical practice, it is very important to ensure the correct and reliable opera- tion of systems at all times. Lutz ${ }^{4,5}$ and the Las Vegas 6,7 phantoms has been reported to check the accuracy and the image quality for the qualitative visual QA checks of patient imaging. ${ }^{8}$

Here in, we report a less subjective approach for automatic daily quality control tests to get better image quality for patient. In a study presently underway at our institute, an integrated EPID-based QA system is being developed, which aims to replace the conventional device-dependent methods for daily and monthly QA tasks. In light of that, this investigation reports on the image quality, relative modulation transfer function, critical frequency and contrast-to-noise ratio obtained.

\section{Methods and Materials}

\section{QC-3 PHANTOM}

The QC-3(Standard Imaging, Middleton, WI) phantom is used to test the image quality from EPIDs. Megavoltage portal images are acquired with the phantom placed on the sur- 
face of an EPID at source-to-detector distance (SDD) $140 \mathrm{~cm}$. The position of phantom can be at different distances such as $160 \mathrm{~cm}, 150 \mathrm{~cm}$ or at isocenter but need to maintain same distance always. A QC-3 phantom was designed for use in the test, which consists of five sets of high-contrast rectangular bars with spatial frequencies of $0.10,0.20,0.25,0.43$, and $0.75 \mathrm{lp} / \mathrm{mm}$ and dimensions of $13.5 \times 11.3 \times 3.6 \mathrm{~cm}^{3}$. A schematic diagram of the QC phantom is shown in Figure 1 (a). The diagram shows the numbered regions of phantom QC-3. The large numbers in the corners are used to for subjective quality control, as they are visible on the image with increasing density as (Number 1 is machined into a lead block to a depth of $1 \mathrm{~mm}$, number 2 to a depth of $2 \mathrm{~mm}$, etc.).

The small numbers indicate the region numbers. Regions 1 5 are bars with different spatial separations, and are used for the analysis of the spatial resolution f5o. Regions 6 - 11 contain blocks of lead or plastic (PVC) with increasing thicknesses. With the EPID located under the patient $\left(0^{\circ}\right.$ in the Varian gantry coordinate systems), the phantom is placed on the top of the EPID detector housing in order to acquire test images. We prefer this location rather than the isocenter to minimize blurring due to the beam penumbra, since the test is intended to monitor the performance of the EPID and should be independent of the linac source size. The phantom is rotated to $45^{\circ}$ relative to the EPID scan lines to prevent aliasing in the image of the bar patterns.

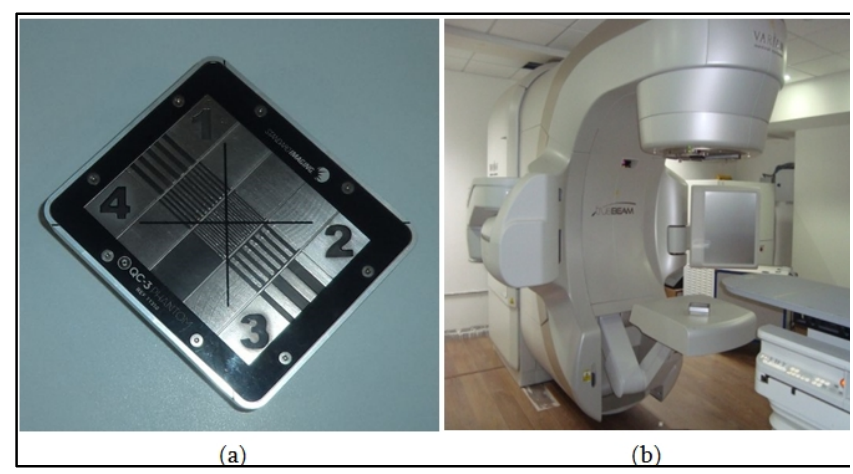

FIG. 1(a) and 1(b): Image of the QC-3 phantom from standard imaging; and phantom position during taking image.

\section{aS1000 EPID}

The Varian aS1000 (Portal Vision, Varian Medical Systems, Palo Alto, CA) is an amorphous silicon flat panel imaging device mounted on a robotic arm. It has an active imaging area of $40 \times 30 \mathrm{~cm}^{2}$ (at an SSD of $105 \mathrm{~cm}$ ). The image matrix is created from an array of $1024 \times 768$ pixels. The maximum frame acquisition rate is 9.574 frames/second, the permitted energy range is $4-25 \mathrm{MV}$, and the permitted dose rates are 50 - $600 \mathrm{MU} / \mathrm{min}$. The detector has four main components. Inside the exterior plastic housing there is a Copper build-up plate, $1 \mathrm{~mm}$ in thickness. This is useful in MV imaging to absorb $\mathrm{x}$-ray photons and emit recoil electrons. It also helps to improve the efficiency of the entire imaging system, by partially shielding the downstream components (including the scintillation screen) from scattered radiation. Underneath this plate lies the phosphor screen. In this EPID it is a Kodak Lanex Fast B scintillating screen, made up of a $0.4 \mathrm{~mm}$ thick Gadolinium Oxysulfide (Gd2O2S: $\mathrm{Tb}$ ) phosphor.

This component absorbs the recoil electrons coming from the Copper plate, and transforms them into visible light. Below the phosphor, there is a $1024 \times 768$ pixel matrix, deposited on a $1 \mathrm{~mm}$ glass substrate. This constitutes the sensitive image forming layer of the photodiode system, and it is $1.5 \mu \mathrm{m}$ thick. Each pixel consists of a Si n-i-photodiode to integrate the incoming light in charge captures and a thin film transistor (TFT) to act as a three-terminal switch for readout. The final major component is the accessory electronics, which drive the TFT switches and read out the charge captures. The gate driver powers the gate lines during the time that the data lines are feeding the accumulated charge to the read-out electronics.

When a voltage is applied to a gate-line, all of the TFTs in that row become transparent and the charge is then transferred to the data lines. Each row is read out in succession, and as one row is read the TFTs in the next row become transparent. External charge sensitive amplifiers capture the charge data. To form one frame of an image, a sequential readout of all of the rows is necessary.

Before each set of EPID images is acquired is it advisable to first calibrate the detector. This can be accomplished by obtaining a dark field and delivering a flood field. The premise is that taking these images will allow for the elimination of background noise and provide a uniform response for imaging. Specifically, the dark field image provides information about background noise, and is obtained by reading out each pixel in the absence of radiation. The resulting image, seen in, is a series of narrow vertical stripes, which result from the photodiode leakage current and varying electrometer offsets. The flood field image, on the other hand, is taken with the entire matrix exposed to a uniform dose. This allows the Portal Vision software to internally correct for individual pixel sensitivities. There is much to say about the acquisition and use of these images inside the Portal Vision software package.

\section{Acquiring QC Images}

The phantom is setup on the EPID at $140 \mathrm{~cm}$, oriented at $45^{\circ}$ to the sagittal plane, as shown in the Figure 1(b). The large number 1 points towards the gantry. Lines and marks on the surface of the phantom assist in lining it up to the central beam line. For routine daily or weekly quality control, it is advisable to mark the surface of the EPID with paint or tape so that placement of the phantom can be done quickly. A stand is available for lateral imaging. Two images of the 
phantom are acquired under identical conditions, preferable during the same irradiation sequence. The obtained images were transferred to the PIPSpro software for analysis.

The analysis program places a region of interest (ROI) over each set of bars as shown in Figure 2. The frequency dependent square wave modulation transfer function (SWMTF) is determined by the method proposed by Droege ${ }^{9}$ and the frequency for $50 \%$ modulation ( $f_{50}$ ) is compared with the predetermined critical frequency $f_{c}$ as a test of system performance.

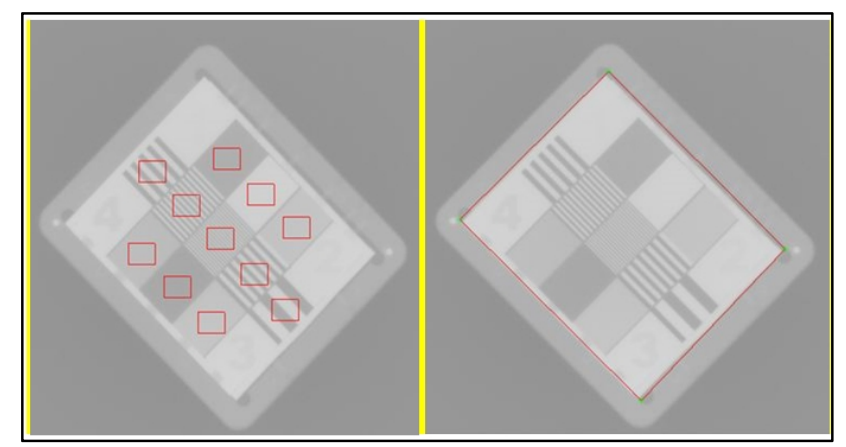

FIG. 2: A portal image of the QC phantom at diagonal orientation obtained with the aS1000 portal imaging system. The ROIs used for the QC test are marked on the image.

TABLE 1: Mean value of $\mathrm{f}_{30}, \mathrm{f}_{40}, \mathrm{f}_{50}$, CNR and noise measured at gantry angle $0^{\circ}$ for both $6 \mathrm{MV}$ and $15 \mathrm{MV}$ phantom is physically displaced relative to the beam center by each of the four directions in a plane orthogonal to the beam.

\begin{tabular}{|c|c|c|c|c|c|c|c|c|c|c|c|}
\hline \multirow[t]{2}{*}{ SL No } & \multirow[t]{2}{*}{ Parameter } & \multicolumn{6}{|c|}{$6 \mathrm{MV}$} & \multicolumn{4}{|c|}{$15 \mathrm{MV}$} \\
\hline & & $f_{30}$ & $f_{40}$ & $f_{50}$ & CNR & Noise & $\mathrm{f}_{30}$ & $f_{40}$ & f50 & CNR & Noise \\
\hline 1 & Without shift & 0.74 & 0.602 & 0.463 & 192.636 & 9.82 & 0.603 & 0.457 & 0.365 & 170.634 & 9.916 \\
\hline 2 & $5^{\circ} \mathrm{Left}$ & 0.739 & 0.599 & 0.46 & 192.376 & 9.754 & 0.6 & 0.452 & 0.362 & 170.35 & 9.868 \\
\hline 3 & $5^{\circ} \mathrm{RT}$ & 0.742 & 0.605 & 0.468 & 189.666 & 9.795 & 0.611 & 0.467 & 0.371 & 167.146 & 9.886 \\
\hline 4 & $1 \mathrm{CM}$ IN & 0.734 & 0.6 & 0.467 & 194.526 & 9.753 & 0.604 & 0.462 & 0.367 & 169.996 & 9.974 \\
\hline 5 & 1CM OUT & 0.745 & 0.605 & 0.464 & 189.15 & 9.918 & 0.605 & 0.456 & 0.366 & 173.23 & 9.748 \\
\hline 6 & 1CM LEFT & 0.741 & 0.603 & 0.465 & 191.173 & 9.846 & 0.606 & 0.46 & 0.367 & 167.544 & 9.947 \\
\hline 7 & 1CM RT & 0.734 & 0.596 & 0.457 & 192.107 & 9.825 & 0.601 & 0.453 & 0.364 & 166.318 & 10.048 \\
\hline
\end{tabular}

TABLE 2: Mean values and standard deviation of $\mathrm{f}_{50}, \mathrm{f}_{40}, \mathrm{f}_{50}, \mathrm{CNR}$ and noise measured at gantry angle $0^{\circ}$ over 40 days $(7$ days calibration period and 33 days test period) for both $6 \mathrm{MV}$ and 15MV Values and standard deviations are rounding up to 3 digits.

\begin{tabular}{|c|c|c|c|c|}
\hline \multirow[t]{2}{*}{ Parameters } & \multicolumn{2}{|c|}{ 6MV Photon energy } & \multicolumn{2}{|c|}{ 15MV Photon energy } \\
\hline & During calibration & During Test period & $\begin{array}{l}\text { During calibration } \\
\text { od }\end{array}$ & During Test peri- \\
\hline$f_{50}$ & $0.463 \pm 0.003$ & $0.464 \pm 0.003$ & $0.365 \pm 0.001$ & $0.366 \pm 0.001$ \\
\hline $\mathrm{f}_{40}$ & $0.602 \pm 0.003$ & $0.602 \pm 0.001$ & $0.457 \pm 0.002$ & $0.458 \pm 0.003$ \\
\hline$f_{30}$ & $0.741 \pm 0.002$ & $0.741 \pm 0.002$ & $0.603 \pm 0.002$ & $0.605 \pm 0.003$ \\
\hline CNR & $192.636 \pm 1.348$ & $191.293 \pm 2.192$ & $170.634 \pm 1.464$ & $170.013 \pm 1.734$ \\
\hline Noise & $9.82 \pm 0.094$ & $9.866 \pm 0.109$ & $9.916 \pm 0.135$ & $9.931 \pm 0.122$ \\
\hline
\end{tabular}

\section{Determination of the RMTF}

The SWMTF of an imaging system is defined as

$$
\operatorname{SWMTF}(\mathrm{f})=\Delta \mathrm{E}(\mathrm{f}) / \Delta \mathrm{E}_{0}
$$

Where $\Delta \mathrm{E}_{0}$ and $\Delta \mathrm{E}$ (f) are the modulations of input to and output from the system. Since we are not interested in the absolute measure of the SWMTF, but only in determine day to-day variations in the system resolution, we use a relative measure ${ }^{10}$ (RMTF) of the SWMTF by calculating:

$$
\operatorname{RTMF}(\mathrm{f})=\Delta \mathrm{E}(\mathrm{f}) / \Delta \mathrm{E}\left(\mathrm{f}_{1}\right)
$$

$$
\text { Or, } \operatorname{RMTF}(\mathrm{f})=\operatorname{MTF}(\mathrm{f}) / \operatorname{MTF}(0.1 \mathrm{LP} / \mathrm{mm})
$$

where, $\Delta \mathrm{E}\left(\mathrm{f}_{1}\right)$ is the output modulation for the lowest frequency.

Usually the output modulation $\Delta \mathrm{E}$ (f) is difficult to obtain from a noisy image; therefore, Droge and Morin ${ }^{11}$ suggest using the relationship between signal amplitude and its variance. For sinusoidal output, $(\Delta \mathrm{E})^{2}$ is proportional to the Variance $(\mathrm{M})^{2}$ within the ROI containing the bar pattern, and the above relation can be written as

$$
\operatorname{RTMF}(\mathrm{f})=\mathrm{M}(\mathrm{f}) / \mathrm{M}\left(\mathrm{f}_{1}\right)
$$

In the presence of random image noise (f) can be obtained by

$$
M^{2}(f)=\sigma_{m}^{2}(f)-\sigma^{2}(f)
$$

where, $\sigma_{\mathrm{m}}^{2}$ (f) and $\sigma^{2}$ (f) are the measured total variance and the variance due to random noise, respectively. The total variance $\sigma_{\mathrm{m}}^{2}(\mathrm{f})$ is obtained by measuring the variance of the pixel in the ROI corresponding the frequency $f$. In order to measure the random noise in an image, a pair of similar images are subtracted, and the standard deviation is obtained from the difference, thus avoiding contributions from fixed pattern noise. In this case, the variance of the subtracted ROI $\left(\sigma_{\text {sub }^{2}}\right)$ will be

$$
\sigma_{\mathrm{sub}^{2}}=\sigma_{1}{ }^{2}+\sigma_{2}{ }^{2}
$$


where, $\sigma_{1}^{2}$ and $\sigma_{2}^{2}$ are the random noise variance of the ROIs for each image. We assume that these two variance are equal and hence;

$$
\sigma_{1}=\sigma=\sigma_{\text {sub }} / \sqrt{ } 2
$$

The variance $\sigma^{2}(\mathrm{f})$ is calculated once using above equation for the set of bars with the highest frequency $(0.75 \mathrm{lp} / \mathrm{mm})$ on the assumption that random noise is same for all ROIs.

\section{Critical Frequency (fso)}

Critical frequency is defined as the spatial resolution corresponding to $50 \%$ RMTF. This value is obtained using a piecewise linear interpolation of the RMTF graph to locate the $50 \%$ relative frequency response. $\left(f_{40}\right)$ is defined as the spatial resolution corresponding to $40 \%$ maximum of the relative modulation transfer function (RMTF). $\mathrm{f}_{30}$ is defined as the spatial resolution corresponding to $30 \%$ maximum of the relative modulation transfer function RMTF.

\section{Contrast-to-Noise Ratio}

A high quality image typically has a large CNR. This can be manipulated by increasing the Contrast, decreasing the noise, or a combination of both. CNR is defined by the following equation:

$$
\mathrm{CNR}=\mathrm{P}_{\text {bright }}-\mathrm{P}_{\mathrm{dark}} / \text { Noise }
$$

where, $\mathrm{P}_{\text {bright }}$ is the average pixel value in the areas receiving the least radiation, $\mathrm{P}_{\text {dark }}$ is the average pixel value in the areas receiving the most radiation dose, and Noise represents the average noise value calculated from the uniformly irradiated regions.

\section{Phantom Alignment}

Electronic portal Imaging systems utilize non-square pixels giving rise to different MTFs in horizontal and vertical directions. By using the diagonal phantom orientation, a combine measure of resolution in both directions could be obtained, as well as reduction in error due to possible changes in ROI size and positioning. ${ }^{9}$ RMTF (f) were exhaustively tested against changes in the ROI size and position. Images are acquired with a $6 \mathrm{MV}$ and $15 \mathrm{MV}$ photon beams when the phantom was physically displaced relative to the beam center by each of the four directions in a plane orthogonal to the beam. Changes in $\mathrm{f}_{50}$ and $\mathrm{CNR}$ were $0.86 \%$ and $0.72 \%$ respectively for $6 \mathrm{MV}$ and $0.27 \%$ and $0.8 \%$ for $15 \mathrm{MV}$. Rotating the phantom by $\pm 5^{\circ}$ from the correct $45^{\circ}$ angle introduced changes in $\mathrm{f}_{50}$ and $\mathrm{CNR}$ of $0.22 \%$ and $0.82 \%$ respectively for $6 \mathrm{MV}$ and $0.27 \%$ and $1.12 \%$ for $15 \mathrm{MV}$.
This displacement and rotations are much larger than any anticipated in normal use of the phantom for routine quality control measurements as mentioned in Table-1. The complete QC procedure was tested by repeating daily for 7 consecutive days. Deliberately moving the ROIs by two pixels (about $1.2 \mathrm{~mm}$ at isocenter) to the left, right, up and down gave change in $\mathrm{f}_{50}$ and $\mathrm{CNR}$ of less than $0.3 \%$ and $1.1 \%$, respectively. Changing the field size and increasing the image acquisition times did not have a significant influence on the measured results.

\section{Results}

Measurements were made daily on the EPID imaging system with dual-energy linear accelerator by using $6 \mathrm{MV}$ and $15 \mathrm{MV}$ photons to 3-5 MU at images for high quality images distance with SDD $140 \mathrm{~cm}$. System performances was monitored during a test period of 1 month and these data were used to determine the mean and standard deviation of $f_{50}, f_{40}$ and $\mathrm{f} 30$.

Before the QC needs to established Base line values for resolution, CNR and noise, after calibration of imager a series of one $\mathrm{QC}$ tests per day over the first one week. "base line" value is shown (Figure 3 ) by the blue line in PIPSpro software. The green area is within the acceptable parameters, the yellow area represents the caution levels, and the red areas represent the reject levels. Suggested values for accept and caution ranges are 5 and 10 percent respectively for all values except noise where 10 and 20 percent is suggested due to the fact that noise tends to fluctuate more than the other parameters.

During the one week calibration the mean values of $f_{50}, f_{40}$ and $f_{30}$ ( \pm standard deviation) were $0.463 \pm 0.003,0.602 \pm$ 0.003 and $0.741 \pm 0.002$ respectively for $6 \mathrm{MV}$. The mean values of $f_{50}, f_{40}$ and $f_{30}( \pm$ standard deviation) were $0.365 \pm$ $0.001,0.457 \pm 0.002$ and $0.603 \pm 0.002$ respectively for $15 \mathrm{MV}$. The mean value of CNR and Noise were $192.636 \pm$ $1.348,9.82 \pm 0.094$ for $6 \mathrm{MV}$ and $170.634 \pm 1.464,9.916 \pm$ 0.135 for $15 \mathrm{MV}$ respectively. Figure 4 and Figure 5 shows $f_{50}$ and CNR a plot of recorded on a daily basis for the as 1000 system which included one month QC data for both high and low energy. The mean values of $f_{50}, f_{40}$ and $f_{30}$ ( \pm standard deviation) were $0.464 \pm 0.003,0.602 \pm 0.001$ and $0.741 \pm$ 0.002 respectively for $6 \mathrm{MV}$. The mean values of $\mathrm{f}_{50}, \mathrm{f}_{40}$ and $\mathrm{f}_{30}$ ( \pm standard deviation) were $0.366 \pm 0.001,0.458 \pm 0.003$ and $0.605 \pm 0.003$ respectively for $15 \mathrm{MV}$. The mean value of CNR and Noise were $191.293 \pm 2.192,9.866 \pm 0.109$ for $6 \mathrm{MV}$ and $170.013 \pm 1.734,9.931 \pm 0.122$ for $15 \mathrm{MV}$ respectively as mentioned in the Table 2. 


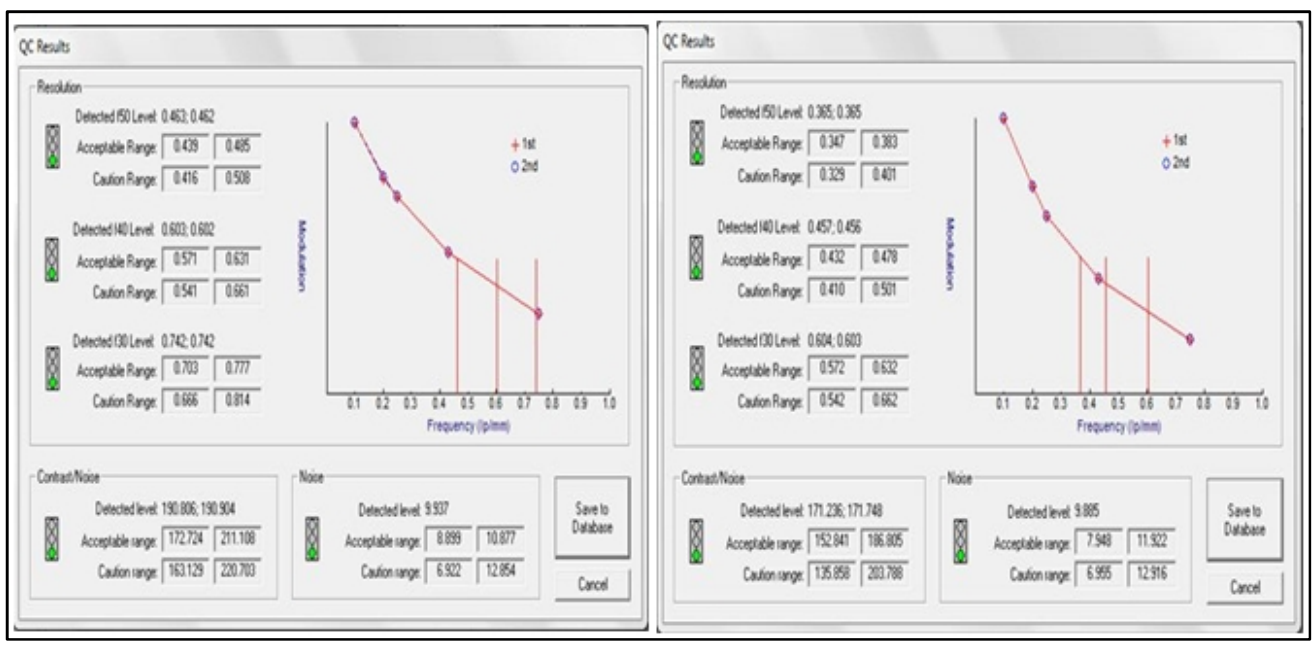

FIG. 3: RMTF curves, created using the PIPSpro Software. The $6 \mathrm{mv}$ curve is in left, and the $15 \mathrm{MV}$ curve is on the right.

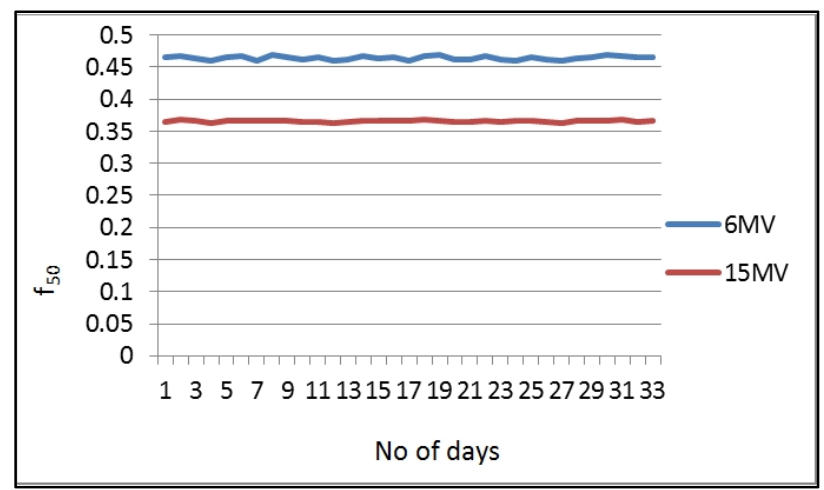

FIG. 4: Plot of f50 recorded on a daily basis for the as 1000 portal imaging system with gantry angle $0^{\circ}$ at $6 \mathrm{MV}$ and $15 \mathrm{MV}$.

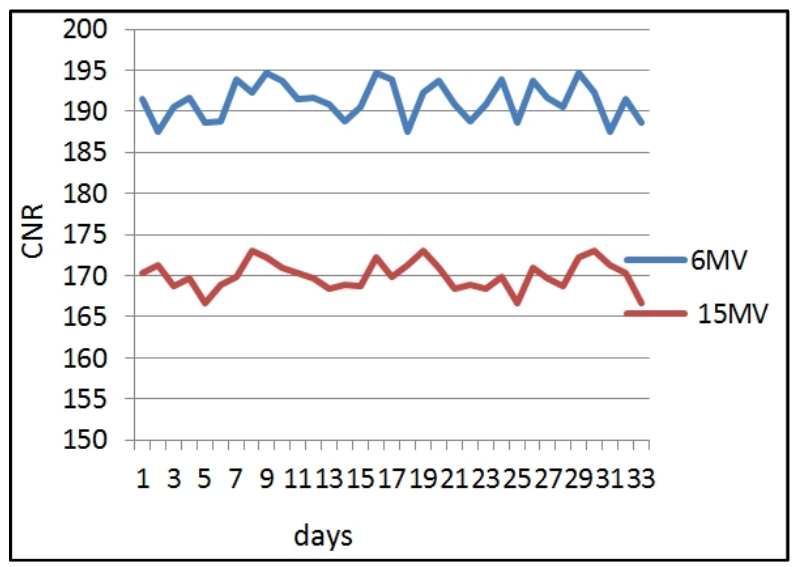

FIG. 5: Plot of CNR recorded on a daily basis for the as 1000 portal imaging system with gantry angle $0^{\circ}$ at $6 \mathrm{MV}$ and $15 \mathrm{MV}$.

It is seen that system resolution at $6 \mathrm{MV}$ is superior to that 15 $\mathrm{MV}$, an effect which has been observed previously and is a result of the larger physical beam penumbra at higher energys ${ }^{12,13,14}$ and increased transmission through the bar patterns by the higher energy photons. It is seen that CNR for $6 \mathrm{MV}$ is higher than that for $15 \mathrm{MV}$. Gantry angle at $90^{\circ}$ also checked during the one week calibration for both $6 \mathrm{MV}$ and $15 \mathrm{MV}$. The mean values of $\mathrm{f}_{50}, \mathrm{f}_{40}$ and $\mathrm{f}_{30}( \pm$ standard deviation) were $0.459 \pm 0.007,0.462 \pm 0.018$ and $0.684 \pm 0.022$ for $6 \mathrm{MV}$ (Figure 6 ). The mean values of $f_{50}, f_{40}$ and $f_{30}( \pm$ standard deviawere $0.421 \pm 0.012,0.531 \pm 0.016$ and $0.645 \pm 0.019$ for The mean value of CNR and Noise were $203.05 \pm 1.923$, \pm 0.085 for $6 \mathrm{MV}$ and $162.528 \pm 1.822,9.399 \pm 0.078$ for respectively (Figure 7).

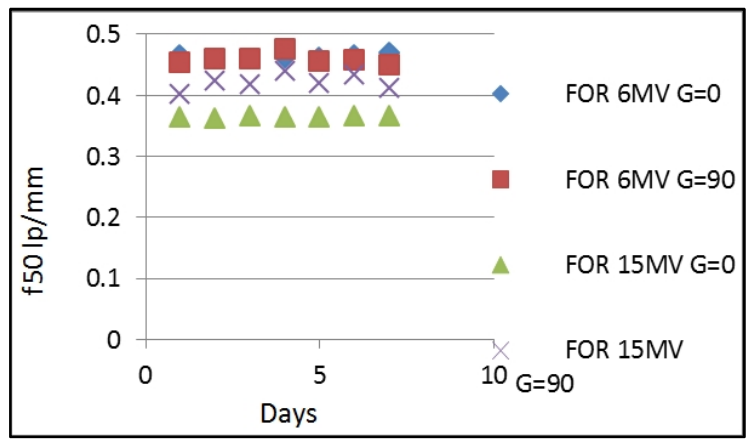

FIG. 6: Plot of f50 recorded on a daily basis for the as 1000 portal imaging system with Gantry Angle $0^{\circ}$ and $90^{\circ}$ at $6 \mathrm{MV}$ and $15 \mathrm{MV}$ for days.

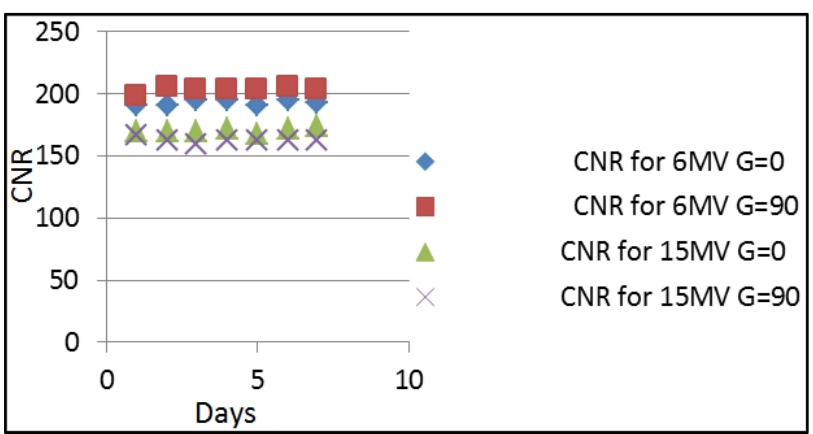

FIG. 7: Plot of f50 recorded on a daily basis for the as1000 portal imaging system with Gantry Angle $0^{\circ}$ and $90^{\circ}$ at $6 \mathrm{MV}$ and $15 \mathrm{MV}$ for 7 days. 


\section{Discussion}

According to Task Group 142 report ${ }^{15}$ : Quality assurance of medical accelerator, spatial resolution, Contrast, uniformity and noise should match to base line. In our case deviation of $\mathrm{f}_{50} \mathrm{CNR}$ and noise were $0.22 \%, 0.70 \%$ and 0.47 for $6 \mathrm{MV}$ and $0.27 \%, 0.36 \%$ and $0.15 \%$ respectively for $15 \mathrm{MV}$ from Base line which is meeting excellently. Quantitatively, specification value for Varian aS1000 f50 was 0.45 for $6 \mathrm{MV}$ and 0.379 for 10-25 MV. In our case spatial Resolution f5o for $6 \mathrm{MV}$ is $0.463 \pm 0.003$ and $0.365 \pm 0.001$ for $15 \mathrm{MV}$ which is very close to given data. Deviation for $6 \mathrm{MV}$ and $15 \mathrm{MV}$ were $2.2 \%$ and $3.4 \%$, respectively. Spatial resolution of $\mathrm{f}_{40}$ and $\mathrm{f}_{30}$ following the $f_{50}$ in proper sequence. The mean values of $f_{50}, f_{40}, f_{30}( \pm$ standard deviation), CNR and noise excellently matching for both during the calibration period and test period for both of $6 \mathrm{MV}$ and $15 \mathrm{MV}$.

\section{Conclusion}

We have established a QC test for portal imaging devices suitable for routine daily use for testing the system for acceptable performance in high contrast spatial resolution and CNR. It is shown that this method provides an automatic, objective, and sensitive measure of the system 's imaging performance and it is useful tool during acceptance testing, commissioning, and routine quality control.

\section{Conflict of interest}

The authors declare that they have no conflicts of interest. The authors alone are responsible for the content and writing of the paper.

\section{References}

1. Kutcher GJ, Coia L, Gillin M, et al. Comprehensive QA for radiation oncology: report of AAPM Radiation Therapy Committee Task Group 40. Med Phys 1994; 21:581-618.

2. Boyer AL, Antonuk L, Fenster A, et al. A review of electronic portal imaging devices (EPIDs). Med Phys 1992; 19:1-16.

3. Verellen D, De Neve W, Van den Heuvel F, et al. On-line portal imaging: image quality defining parameters for pelvic fields--a clinical evaluation. Int J Radiat Oncol Biol Phys 1993; 27:945-52.

4. Lutz WR, Bjarngard BE. A test object for evaluation of portal films. Int $J$ Radiat Oncol Biol Phys 1985; 11:631-4.

5. Leszczynski KW, Shalev S. Digital contrast enhancement for online portal imaging. Med Biol Eng Comput 1989; 27:507-12.

6. Wong JW, Cheng AY, Binns WR, et al. Development of a second-generation fiber-optic on-line image verification system. Int I Radiat Oncol Biol Phys 1993; 26:311-20.

7. Herman MG, Abrams RA, Mayer RR. Clinical use of on-line portal imaging for daily patient treatment verification. Int J Radiat Oncol Biol Phys 1994; 28:1017-23.

8. Murthy KK, Al-Rahbi Z, Sivakumar SS, et al. Verification of set up errors in external beam radiation therapy using electron portal imaging. Med Phys 2008; 33:49-53.

9. Droege RT. A practical method to routinely monitor resolution in digital images. Med Phys 1983; 10:337-43.

10. Arimura H, Kubota H, Matsumoto M, et al. Wiener spectra of quantum mottle and the squares of modulation transfer function. Phys Med Biol 1999; 44:1337-52.

11. Droege RT, Morin RL. A practical method to measure the MTF of CT scanners. Med Phys 1982; 9:758-60.

12. Wowk B, Radcliffe T, Leszczynski KW, Shalev S, Rajapakshe R. Optimization of metal/phosphor screens for on-line portal imaging. Med Phys 1994; 21:227-35.

13. Munro P, Rawlinson JA, Fenster A. Therapy imaging: a signal-to-noise analysis of a fluoroscopic imaging system for radiotherapy localization. Med Phys 1990; 17:763-72.

14. Rajapakshe R, Luchka K, Shalev S. A quality control test for electronic portal imaging devices. Med Phys 1996; 23:1237-44.

15. Klein EE, Hanley J, Bayouth J, et al. Task Group 142 report: quality assurance of medical accelerators. Med Phys 2009; 36:4197-212. 